\title{
Synthesis of 5-aryl-1-carboxyphenyl-3-(3-coumarinyl)-2-pyrazolines
}

\author{
Albert Lévai ${ }^{a} *$ and József Jekőb \\ ${ }^{a}$ Department of Organic Chemistry, University of Debrecen, P.O.Box 20, H 4010 Debrecen, \\ Hungary \\ ${ }^{b}$ Department of Chemistry, College of Nyíregyháza, Sóstói u. 31/b, 4400 Nyíregyháza, Hungary \\ E-mail: alevai@puma.unideb.hu
}

\section{Dedicated to Professor Henk van der Plas on the occasion of his $80^{\text {th }}$ birthday}

\begin{abstract}
1-(2-Carboxyphenyl)- and 1-(4-carboxyphenyl)-2-pyrazolines have been synthesized by treatment of appropriate 3-aryl-1-(3-coumarinyl)propen-1-ones with (2-carboxyphenyl)hydrazine and (4-carboxyphenyl)hydrazine in hot acetic acid. The structures of all new 2-pyrazolines have been elucidated by microanalyses, IR, ${ }^{1} \mathrm{H}$ - and ${ }^{13} \mathrm{C}-\mathrm{NMR}$ - spectroscopic measurements.
\end{abstract}

Keywords: 3-aryl-1-(3-coumarinyl)propen-1-ones, (2-carboxyphenyl)hydrazine, (4-carboxyhenyl)hydrazine, 2-pyrazolines

\section{Introduction}

Coumarins are well known, naturally occurring oxygen- heterocyclic compounds isolated from various plants. $^{1,2}$ Owing to their diverse bioactivities, viz. antibacterial and antifungal, ${ }^{3}$ anticoagulant, 4,5 and other effects, natural, semi-synthetic and synthetic coumarins are useful substances in drug research. Some coumarin derivatives can be utilized beneficially for the synthesis of valuable heterocyclic ring systems. 3-Cinnamoylcoumarins obtained by the reaction of 3-acetylcoumarins with aromatic aldehydes ${ }^{5-11}$ proved to be especially important compounds for this purpose. Pyridines, ${ }^{5}$ isoxazolines, ${ }^{11}$ 1,5-benzodiazepines, ${ }^{12}$ 1,5-benzothiazepines, ${ }^{13-16}$ pyrazolines $^{17}$ and other nitrogen-containing heterocyclic compounds were synthesized using 3cinnamoyl coumarins as starting materials.

Pyrazolines are important nitrogen-containing heterocyclic compounds and some of them possess valuable biological activities, e.g., central nervous system-, ${ }^{18}$ immunosuppressive-,${ }^{19}$ and antimicrobial $^{20}$ activities. 2-Pyrazolines are the most popular and frequently studied pyrazoline isomers. $^{21-23}$ Reactions of $\alpha, \beta$-unsaturated aldehydes and ketones with hydrazines offer a versatile procedure for their preparation. ${ }^{23}$ As a continuation of our previous studies in this 
field, ${ }^{17,24-31}$ herein we report on the synthesis of 5-aryl-1-carboxyphenyl-3-(3-coumarinyl)-2pyrazolines.

\section{Results and Discussion}

For the synthesis of 2-pyrazolines by the reaction of $\alpha, \beta$-unsaturated ketones with hydrazines, hydrazine or phenylhydrazine were used as nitrogen- donor reagents in the most cases. ${ }^{22,23}$ For the utilization of some other hydrazine derivatives, viz. 4-fluorophenylhydrazine, ${ }^{32,33}$ 4-chlorophenylhydrazine, ${ }^{34}$ semicarbazide and thiosemicarbazide, ${ }^{25,35-37}$ 2-hydrazinopyridine and 4-hydrazinophthalazine ${ }^{38}$ only sporadic examples have been published in the literature. In the course of our previous studies on the synthesis of 2-pyrazolines, we had prepared 1-(2carboxyphenyl)- and 1-(4-carboxyphenyl)-2-pyrazolines by the reaction of $\alpha, \beta$-unsaturated ketones with (2-carboxyphenyl)- and (4-carboxyphenyl)hydrazine. ${ }^{39-41}$

We have synthesized 5-aryl-3-(3-coumarinyl)-1-phenyl-2-pyrazolines by the reaction of 3aryl-1-(3-coumarinyl)propen-1-ones and phenylhydrazine in hot pyridine. ${ }^{17}$ However, under these reaction conditions, intractable dark brown mixtures were obtained when these $\alpha, \beta$-unsaturated ketones were reacted either with (2-carboxyphenyl)hydrazine or with (4-carboxyphenyl)hydrazine. In our previous studies, 1-(2-carboxyphenyl)- and 1-(4-carboxyphenyl)-2-pyrazolines were successfully synthesized by the reaction of $\alpha, \beta$ unsaturated ketones with (2-carboxyphenyl)- and (4-carboxyphenyl)-hydrazine in hot acetic acid. $^{39,40}$ For this reason, in our present study, 3-aryl-1-(3-coumarinyl)propen-1-ones 1-7 were allowed to react with (2-carboxyphenyl)- and (4-carboxyphenyl)hydrazine in hot acetic acid, and the 2-pyrazolines 8-21 (Scheme 1) were obtained in medium- to good yields (64-75\%). The electronic character of the $p$ - substituent in the aromatic ring $\left(\mathrm{R}^{1}\right)$ and the place of the carboxyl group $\left(\mathrm{R}^{2}\right.$ and $\mathrm{R}^{3}$ ) were without influence on the course or the yield of the reaction.

The structures of all new compounds were elucidated by elemental analyses and combined spectroscopic techniques. Elemental analyses unambiguously proved the elemental composition of all new compounds 8-21. In their IR spectra, a characteristic lactone carbonyl band at around $1730 \mathrm{~cm}^{-1}$ proves the presence of the coumarin moiety. An intense carboxyl band was assigned only in the case of the 1-(4-carboxyphenyl)-2-pyrazolines $\mathbf{1 5 - 2 1}$ between 1670 and $1680 \mathrm{~cm}^{-1}$. Owing to the hydrogen-bond formation with the neighboring nitrogen atom, this carboxyl band is absent from the IR spectra of the 1-(2-carboxyphenyl)-2-pyrazolines 8-14. A $\mathrm{C}=\mathrm{N}$ band at around $1600 \mathrm{~cm}^{-1}$ is in harmony with the 2-pyrazoline skeleton. In the ${ }^{1} \mathrm{H}-\mathrm{NMR}$ spectra of compounds 8-21 the protons attached to the C-4 and C-5 carbon atoms of the five-membered ring (Scheme 1) gave an ABX spin system. Chemical shifts and the coupling constant values ( $c f$. Experimental Section) unequivocally prove the 2-pyrazoline structure. ${ }^{22,23}$ Because of the strong hydrogen bond in the case of the 1-(2-carboxyphenyl)-2-pyrazolines 8-14 no proton signal assigned to the carboxyl group could be detected. While in the ${ }^{1} \mathrm{H}$ - NMR spectra of the 1-(4carboxyphenyl)-2-pyrazolines 15-21 a distinct singlet signal belonging to the carboxyl group was 
found at around 12.20-12.50 ppm. In their ${ }^{13} \mathrm{C}-\mathrm{NMR}$ spectra, the chemical shift values of carbon atoms C-3 (152-153 ppm), C-4 (about $45 \mathrm{ppm}$ ) and C-5 (around $62 \mathrm{ppm}$ ) corroborate the 2-pyrazoline structure deduced from the IR and ${ }^{1} \mathrm{H}-\mathrm{NMR}$ spectroscopic data.<smiles>[R]c1ccc(/C=C/C(=O)c2cc3ccccc3oc2=O)cc1</smiles>

1-7

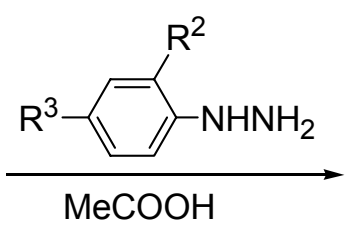

1,15: $R^{1}=R^{2}=H, R^{3}=\mathrm{COOH}$

2,16: $R^{1}=M e, R^{2}=H, R^{3}=\mathrm{COOH}$

3,17: $\mathrm{R}^{1}=\mathrm{iPr}, \mathrm{R}^{2}=\mathrm{H}, \mathrm{R}^{3}=\mathrm{COOH}$

4,18: $\mathrm{R}^{1}=\mathrm{MeO}, \mathrm{R}^{2}=\mathrm{H}, \mathrm{R}^{3}=\mathrm{COOH}$

5,19: $R^{1}=F, R^{2}=H, R^{3}=\mathrm{COOH}$

6,20: $\mathrm{R}^{1}=\mathrm{Cl}, \mathrm{R}^{2}=\mathrm{H}, \mathrm{R}^{3}=\mathrm{COOH}$

7,21: $R^{1}=B r, R^{2}=H, R^{3}=\mathrm{COOH}$

\section{Scheme 1}

In conclusion, we have introduced a simple and convenient protocol for the synthesis of hitherto unknown group of 2-pyrazolines bearing a 3-coumarinyl moiety and a carboxyl group. Since both the coumarins and the 2-pyrazolines were found to possess important bioactivities, these new 2-pyrazoline derivatives may be beneficially utilized in the drug research.

\section{Experimental Section}

General Procedures. Melting points were determined on a Kofler hot-stage apparatus and are uncorrected. ${ }^{1} \mathrm{H}$ - and ${ }^{13} \mathrm{C}$ - NMR spectra were measured with a Bruker WP $200 \mathrm{SY}$ spectrometer at 200/50 MHz in DMSO-d 6 (internal standard TMS, $\delta=0.0 \mathrm{ppm}$ ) at ambient temperature (ca 20 $\left.{ }^{\circ} \mathrm{C}\right)$. The IR spectra were obtained with a Perkin-Elmer 16 PC instrument. Elemental analyses $(\mathrm{C}$, $\mathrm{H}, \mathrm{N})$ were measured in-house with a Carlo Erba 1106 EA instrument. TLC was performed on Kiselgel $60 \mathrm{~F}_{254}$ (Merck) layer using toluene:ethyl acetate $(4: 1 \mathrm{v} / \mathrm{v})$ as eluent. Starting materials 1-7 were synthesized according to known procedures. ${ }^{5-11}$ 
General method for the preparation of 2-pyrazolines 8-21. A mixture of the appropriate 3aryl-1-(3-coumarinyl)propen-1-one (1-7, $5.0 \mathrm{mmol})$, (2-carboxyphenyl)hydrazine (15.0 mmol) or (4-carboxyphenyl)hydrazine $(15.0 \mathrm{mmol})$ and acetic acic $(100 \mathrm{~mL})$ was refluxed for $6 \mathrm{~h}$, then poured into water. The precipitate was separated by filtration, washed with water, dried and purified by column chromatography on Kieselgel 60 columns using toluene:ethyl acetate (4:1 $v / v$ ) as eluent to obtain 2-pyrazolines 8-21 (Scheme 1).

1-(2-Carboxyphenyl)-3-(3-coumarinyl)-5-phenyl-2-pyrazoline (8). Isolated as orange needles in $67 \%$ yield, mp $180-182{ }^{\circ} \mathrm{C}$ (methanol); ${ }^{1} \mathrm{H}-\mathrm{NMR}\left(\delta, \mathrm{DMSO}_{6}\right): 3.23(1 \mathrm{H}, \mathrm{dd}, \mathrm{J}=6.8,17.6$ $\left.\mathrm{Hz}, 4-\mathrm{H}_{\text {trans }}\right), 3.98$ (1H, dd, J = 12.1, $\left.17.6 \mathrm{~Hz}, 4-\mathrm{H}_{\text {cis }}\right), 5.54(1 \mathrm{H}, \mathrm{dd}, \mathrm{J}=6.8,12.1 \mathrm{~Hz}, 3-\mathrm{H}), 6.78-$ $7.82\left(\mathrm{~m}, 13\right.$ arom. H), $8.51\left(1 \mathrm{H}, \mathrm{s}, 4{ }^{\prime}-\mathrm{H}\right) ;{ }^{13} \mathrm{C}-\mathrm{NMR}\left(\delta, \mathrm{DMSO}-\mathrm{d}_{6}\right): 44.5,63.0,113.2,115.8$, $116.0,119.1,119.9,124.7,125.7,127.4,128.7,128.8,128.9,129.1,131.8,142.2,143.5,152.9$, 158.1; IR ( $\left.\mathrm{cm}^{-1}\right)$ : 1730, 1599, 1564, 1498, 1455, 1321, 1276, 1120, 970, 876, 747, 698; Anal. Calcd. for $\mathrm{C}_{25} \mathrm{H}_{18} \mathrm{~N}_{2} \mathrm{O}_{4}$ : C, 73.16; H, 4.42; N, 6.82. Found: C, 73.25; H, 4.46; N, 6.89\%.

1-(2-Carboxyphenyl)-3-(3-coumarinyl)-5-(4-methylphenyl)-2-pyrazoline (9). Prepared as orange needles in $64 \%$ yield, mp $166-167{ }^{\circ} \mathrm{C}$ (methanol); $\left.{ }^{1} \mathrm{H}-\mathrm{NMR}(\delta \text {, DMSO-d })_{6}\right): 2.28(3 \mathrm{H}, \mathrm{s}$, Me), 3.20 (1H, dd, J = 7.1, $\left.17.4 \mathrm{~Hz}, 4-\mathrm{H}_{\text {trans }}\right), 3.96\left(1 \mathrm{H}, \mathrm{dd}, \mathrm{J}=11.8,17.4 \mathrm{~Hz}, 4-\mathrm{H}_{\text {cis }}\right), 5.48(1 \mathrm{H}$, $\mathrm{dd}, \mathrm{J}=7.1,11.8 \mathrm{~Hz}, 3-\mathrm{H}), 6.74-7.83$ (m, 12 arom. H), 8.48 (1H, s, 4'-H); ${ }^{13} \mathrm{C}-\mathrm{NMR}(\delta$, DMSO$\left.\mathrm{d}_{6}\right)$ : 20.6, 44.5, 62.8, 113.3, 115.8, 119.1, 119.9, 124.7, 125.7, 128.7, 128.8, 129.5, 131.8, 136.6, 138.3, 139.2, 143.1, 143.5, 152.8, 158.1; IR ( $\left.\mathrm{cm}^{-1}\right)$ : 1730, 1597, 1497, 1390, 1323, 1131, 867, 813, 749, 690; Anal. Calcd for $\mathrm{C}_{26} \mathrm{H}_{20} \mathrm{~N}_{2} \mathrm{O}_{4}$ : C, 73.57; H, 4.75; N, 6.59. Found: C, 73.68; H, 4.70; N, 6.65\%.

1-(2-Carboxyphenyl)-3-(3-coumarinyl)-5-(4-isopropylphenyl)-2-pyrazoline (10). Orange needles, prepared in 68\% yield, mp $174-176{ }^{\circ} \mathrm{C}$ (methanol); ${ }^{1} \mathrm{H}-\mathrm{NMR}\left(\delta, \mathrm{DMSO}-\mathrm{d}_{6}\right): 1.20(6 \mathrm{H}$,

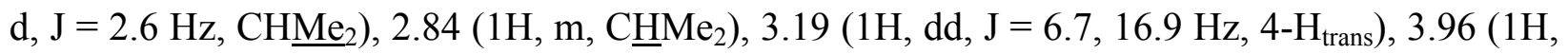
$\left.\mathrm{dd}, \mathrm{J}=12.0,16.9 \mathrm{~Hz}, 4-\mathrm{H}_{\mathrm{cis}}\right), 5.50(1 \mathrm{H}, \mathrm{dd}, \mathrm{J}=6.7,12.0 \mathrm{~Hz}, 3-\mathrm{H}), 6.82-7.80$ (m, 12 arom. H), $8.50\left(1 \mathrm{H}, \mathrm{s}, 4^{\prime}-\mathrm{H}\right) ;{ }^{13} \mathrm{C}-\mathrm{NMR}\left(\delta, \mathrm{DMSO}_{\mathrm{d}}\right)$ : 23.7, 32.9, 44.5, 62.6, 113.2, 115.8, 119.1, 119.2, $124.7,125.6,126.6,126.9,128.3,128.7,128.9,129.1,131.8,138.4,139.6,143.1 ., 143.5,147.5$, 152.8, 158.1; IR (cm $\left.{ }^{-1}\right): 1728,1599,1564,1498,1455,1384,1320,1275,1120,1071,972,879$, 819, 751, 691; Anal. Calcd. for $\mathrm{C}_{28} \mathrm{H}_{24} \mathrm{~N}_{2} \mathrm{O}_{4}$ : C, 74.32; H, 5.35; N, 6.19. Found: C, 74.23; H, $5.40 ; \mathrm{N}, 6.25 \%$.

1-(2-Carboxyphenyl)-3-(3-coumarinyl)-5-(4-methoxyphenyl)-2-pyrazoline (11). Obtained as red needles in $72 \%$ yield, mp $164-165{ }^{\circ} \mathrm{C}$ (methanol); ${ }^{1} \mathrm{H}-\mathrm{NMR}\left(\delta, \mathrm{DMSO}-\mathrm{d}_{6}\right): 3.19(1 \mathrm{H}, \mathrm{dd}, \mathrm{J}=$ 6.2, $\left.17.9 \mathrm{~Hz}, 4-\mathrm{H}_{\text {trans }}\right), 3.62(3 \mathrm{H}, \mathrm{s}, \mathrm{MeO}), 3.94\left(1 \mathrm{H}, \mathrm{dd}, \mathrm{J}=11.6,17.9 \mathrm{~Hz}, 4-\mathrm{H}_{\mathrm{cis}}\right), 5.46(1 \mathrm{H}, \mathrm{dd}$, $\mathrm{J}=6.2,11.6 \mathrm{~Hz}, 3-\mathrm{H}), 6.73-7.81\left(\mathrm{~m}, 12\right.$ arom. H), $8.46\left(1 \mathrm{H}, \mathrm{s}, 4{ }^{\prime}-\mathrm{H}\right) ;{ }^{13} \mathrm{C}-\mathrm{NMR}(\delta$, DMSO-d 6 ): $44.5,54.9,62.5,113.3,114.3,115.8,119.1,119.2,120.0,124.7,124.8,127.0,128.7,128.8$, 131.8, 134.1, 138.3, 143.1, 143.5, 152.8, 158.5; IR (cm $\left.{ }^{-1}\right): 1729,1598,1564,1498,1456,1391$, $1323,1246,1173,1126,1033,971,923,867,828,750,690$; Anal. Calcd. for $\mathrm{C}_{26} \mathrm{H}_{20} \mathrm{~N}_{2} \mathrm{O}_{5}$ : C, 70.90; H, 4.58; N, 6.36. Found: C, 70.82; H, 4.64; N, 6.29\%. 
1-(2-Carboxyphenyl)-3-(3-coumarinyl)-5-(4-fluorophenyl)-2-pyrazoline (12). Isolated as red needles in $74 \%$ yield, mp 139-140 ${ }^{\circ} \mathrm{C}$ (methanol); ${ }^{1} \mathrm{H}-\mathrm{NMR}\left(\delta, \mathrm{DMSO}-\mathrm{d}_{6}\right): 3.22(1 \mathrm{H}, \mathrm{dd}, \mathrm{J}=6.6$, $\left.17.7 \mathrm{~Hz}, 4-\mathrm{H}_{\text {trans }}\right), 4.00\left(1 \mathrm{H}, \mathrm{dd}, \mathrm{J}=12.4,17.7 \mathrm{~Hz}, 4-\mathrm{H}_{\text {cis }}\right), 5.56(1 \mathrm{H}, \mathrm{dd}, \mathrm{J}=6.6,12.4 \mathrm{~Hz}, 3-\mathrm{H})$, 6.78-7.84 (m, 12 arom. H), $8.50\left(1 \mathrm{H}, \mathrm{s}, 4{ }^{\prime}-\mathrm{H}\right) ;{ }^{13} \mathrm{CNMR}\left(\delta, \mathrm{DMSO}_{6}\right)$ : 44.4, 62.3, 113.3, 115.6, $115.8,116.0,119.1,119.3,119.8,124.7,127.8,127.9,128.7,128.9,131.9,138.3,138.5,143.2$, 143.4, 152.9, 158.1; IR (cm $\left.{ }^{-1}\right): 1730,1602,1565,1499,1456,1390,1324,1276,1223,972,833$, 751, 690; Anal. Calcd. for $\mathrm{C}_{25} \mathrm{H}_{17} \mathrm{FN}_{2} \mathrm{O}_{4}$ : C, 70.09; H, 4.01; N, 6.54. Found: C, 70.16; H, 4.06; $\mathrm{N}, 6.46 \%$.

1-(2-Carboxyphenyl)-5-(4-chlorophenyl)-3-(3-coumarinyl)-2-pyrazoline (13). Obtained as red needles in $75 \%$ yield, mp $188-190{ }^{\circ} \mathrm{C}$ (methanol); ${ }^{1} \mathrm{H}-\mathrm{NMR}\left(\delta, \mathrm{DMSO}-\mathrm{d}_{6}\right): 3.24(1 \mathrm{H}, \mathrm{dd}, \mathrm{J}=$ 6.1, $\left.17.9 \mathrm{~Hz}, 4-\mathrm{H}_{\text {trans }}\right), 4.01\left(1 \mathrm{H}, \mathrm{dd}, \mathrm{J}=12.5,17.9 \mathrm{~Hz}, 4-\mathrm{H}_{\mathrm{cis}}\right), 5.51$ (1H, dd, J = 6.1, $12.5 \mathrm{~Hz}, 3-$ $\mathrm{H}), 6.80-7.84$ (m, 12 arom. H), $8.47\left(1 \mathrm{H}, \mathrm{s}, 4{ }^{\prime}-\mathrm{H}\right) ;{ }^{13} \mathrm{C}-\mathrm{NMR}\left(\delta, \mathrm{DMSO}-\mathrm{d}_{6}\right): 44.3,62.3,113.2$, $115.9,119.4,119.8,124.7,127.8,128.7,131.9,132.0,138.6,141.1,143.3,152.9,158.1$; IR $\left(\mathrm{cm}^{-}\right.$ $\left.{ }^{1}\right): 1729,1599,1565,1498,1456,1383,1320,1252,1120,972,880,822$, 751, 692; Anal. Calcd. for $\mathrm{C}_{25} \mathrm{H}_{17} \mathrm{ClN}_{2} \mathrm{O}_{4}$ : C, 67.49; H, 3.85; N, 6.29. Found: C, 67.58; H, 3.80; N, 6.35\%.

5-(4-Bromophenyl)-1-(2-carboxyphenyl)-3-(3-coumarinyl)-2-pyrazoline (14). Prepared as red needles in 67\% yield, mp 199-201 ${ }^{\circ} \mathrm{C}$ (methanol); ${ }^{1} \mathrm{H}-\mathrm{NMR}\left(\delta, \mathrm{DMSO}-\mathrm{d}_{6}\right): 3.20(1 \mathrm{H}, \mathrm{dd}, \mathrm{J}=6.8$, $\left.17.9 \mathrm{~Hz}, 4-\mathrm{H}_{\text {trans }}\right), 3.98\left(1 \mathrm{H}, \mathrm{dd}, \mathrm{J}=12.4,17.9 \mathrm{~Hz}, 4-\mathrm{H}_{\text {cis }}\right), 5.52(1 \mathrm{H}, \mathrm{dd}, \mathrm{J}=6.8,12.4 \mathrm{~Hz}, 3-\mathrm{H})$, 6.84-7.86 (m, 12 arom. H), 8.48 (1H, s, $\left.4^{\prime}-\mathrm{H}\right) ;{ }^{13} \mathrm{C}-\mathrm{NMR}\left(\delta, \mathrm{DMSO}_{\mathrm{d}}\right.$ ): 44.2, 62.3, 113.2, 115.9 , $116.0,117.4,119.1,119.4,119.8,120.5,124.7,125.3,128.1,128.7,128.9,131.9,132.6,138.6$, 141.5, 143.3, 152.9, 158.1; IR ( $\left.\mathrm{cm}^{-1}\right): 1731,1597,1565,1497,1456,1388,1322,1244,1120$, 1028, 971, 922, 827, 754, 689; Anal. Calcd. for $\mathrm{C}_{25} \mathrm{H}_{17} \mathrm{BrN}_{2} \mathrm{O}_{4}: \mathrm{C}, 61.36 ; \mathrm{H}, 3.50 ; \mathrm{N}, 5.72$. Found: C, 61.28; H, 3.54; N, 5.79\%.

1-(4-Carboxyphenyl)-3-(3-coumarinyl)-5-phenyl-2-pyrazoline (15). Obtained as orange needles in 71\% yield, mp 270-271 ${ }^{\circ} \mathrm{C}$ (methanol); ${ }^{1} \mathrm{H}-\mathrm{NMR}\left(\delta, \mathrm{DMSO}-\mathrm{d}_{6}\right): 3.24(1 \mathrm{H}, \mathrm{dd}, \mathrm{J}=5.4$, $\left.17.8 \mathrm{~Hz}, 4-\mathrm{H}_{\text {trans }}\right), 4.04\left(1 \mathrm{H}, \mathrm{dd}, \mathrm{J}=12.0,17.8 \mathrm{~Hz}, 4-\mathrm{H}_{\mathrm{cis}}\right), 5.50(1 \mathrm{H}, \mathrm{dd}, \mathrm{J}=5.4,12.0 \mathrm{~Hz}, 3-\mathrm{H})$, 7.05-8.10 (m, 13 arom. H), $8.46\left(1 \mathrm{H}, \mathrm{s}, 4{ }^{\prime}-\mathrm{H}\right), 12.18(1 \mathrm{H}, \mathrm{s}, \mathrm{COOH}) ;{ }^{13} \mathrm{C}-\mathrm{NMR}(\delta$, DMSO-d 6 ): 44.6, 62.6, 112.2, 115.7, 117.2, 119.1, 120.5, 125.4, 126.3, 127.9, 128.6, 128.8, 130.3, 130.8, $131.9,132.3,136.4,139.2,141.0,142.8,143.4,145.2,150.1,158.0,167.2 ;$ IR $\left(\mathrm{cm}^{-1}\right): 1733$, 1676, 1599, 1522, 1484, 1455, 1417, 1372, 1317, 1288, 1176, 1132, 1105, 1078, 874, 756, 697; Anal. Calcd. for $\mathrm{C}_{25} \mathrm{H}_{18} \mathrm{~N}_{2} \mathrm{O}_{4}$ : C, 73.16; H, 4.42; N, 6.82. Found: C, 73.08; H, 4.37; N, 6.88\%.

1-(4-Carboxyphenyl)-3-(3-coumarinyl)-5-(4-methylphenyl)-2-pyrazoline (16). Prepared as orange needles in 69\% yield, mp 298-300 ${ }^{\circ} \mathrm{C}$ (methanol); ${ }^{1} \mathrm{H}-\mathrm{NMR}\left(\delta, \mathrm{DMSO}-\mathrm{d}_{6}\right): 2.28(3 \mathrm{H}, \mathrm{s}$, $\mathrm{Me}), 3.28\left(1 \mathrm{H}, \mathrm{dd}, \mathrm{J}=5.8,17.6 \mathrm{~Hz}, 4-\mathrm{H}_{\text {trans }}\right), 4.02\left(1 \mathrm{H}, \mathrm{dd}, \mathrm{J}=11.8,17.6 \mathrm{~Hz}, 4-\mathrm{H}_{\text {cis }}\right), 5.51(1 \mathrm{H}$, dd, J = 5.8, $11.8 \mathrm{~Hz}, 3-\mathrm{H}), 7.08-8.12$ (m, 12 arom. H), 8.57 (1H, s, 4'-H), 12.44 (1H, s, COOH);

${ }^{13} \mathrm{C}(\delta$, DMSO-d 6$): 20.6,44.6,62.2,112.4,115.9,119.1,119.6,120.5,124.8,125.6,126.6$, $129.6,130.7,131.8,132.2,136.8,139.6,145.8,146.5,153.0,158.0,167.1 ;$ IR $\left(\mathrm{cm}^{-1}\right): 1730$, $1671,1601,1517,1485,1420,1310,1292$, 1222, 1175, 1131, 975, 870, 761; Anal. Calcd. for $\mathrm{C}_{26} \mathrm{H}_{20} \mathrm{~N}_{2} \mathrm{O}_{4}$ : C, 73.57; H, 4.75; N, 6.59. Found: C, 73.49; H, 4.78; N, 6.53\%. 
1-(4-Carboxyphenyl)-3-(3-coumarinyl)-5-(4-isopropylphenyl)-2-pyrazoline (17). Isolated as orange needles in 70\% yield, mp 270-271 ${ }^{\circ} \mathrm{C}$ (methanol); ${ }^{1} \mathrm{H}-\mathrm{NMR}\left(\delta, \mathrm{DMSO}-\mathrm{d}_{6}\right): 1.20(6 \mathrm{H}, \mathrm{d}, \mathrm{J}$ $=2.4 \mathrm{~Hz}, \mathrm{CHMe} 2), 2.84\left(1 \mathrm{H}, \mathrm{m}, \underline{\mathrm{CHMe}}_{2}\right), 3.28\left(1 \mathrm{H}, \mathrm{dd}, \mathrm{J}=6.1,17.6 \mathrm{~Hz}, 4-\mathrm{H}_{\text {trans }}\right), 4.04(1 \mathrm{H}, \mathrm{dd}$, $\left.\mathrm{J}=11.9,17.6 \mathrm{~Hz}, 4-\mathrm{H}_{\mathrm{cis}}\right), 5.60(1 \mathrm{H}, \mathrm{dd}, \mathrm{J}=6.1,11.9 \mathrm{~Hz}, 3-\mathrm{H}), 7.10-8.14$ (m, 12 arom. H), 8.52 $\left(1 \mathrm{H}, \mathrm{s}, 4{ }^{\prime}-\mathrm{H}\right), 12.34(1 \mathrm{H}, \mathrm{s}, \mathrm{COOH}) ;{ }^{13} \mathrm{C}-\mathrm{NMR}\left(\delta, \mathrm{DMSO}_{\mathrm{d}}\right): 23.7,33.0,44.6,62.0,112.3$, $115.9,116.1,117.4,119.2,124.2,124.8,125.3,125.6,126.7,128.9,129.1,130.8,131.1,132.6$, 139.7, 142.9, 143.5, 153.1, 158.0, 167.1; IR $\left(\mathrm{cm}^{-1}\right): 1730,1677,1600,1570,1518,1457,1416$, 1319, 1289, 1204, 1174, 875, 842, 750; Anal. Calcd. for $\mathrm{C}_{28} \mathrm{H}_{24} \mathrm{~N}_{2} \mathrm{O}_{4}$ : C, 74.32; H, 5.35; N, 6.19. Found: C, 74.25; H, 5.31; N, 6.26\%.

1-(4-Carboxyphenyl)-3-(3-coumarinyl)-5-(4-methoxyphenyl)-2-pyrazoline (18). Prepared as orange needles in $73 \%$ yield, mp 304-305 ${ }^{\circ} \mathrm{C}$ (methanol); ${ }^{1} \mathrm{H}-\mathrm{NMR}\left(\delta, \mathrm{DMSO}-\mathrm{d}_{6}\right): 3.26(1 \mathrm{H}$, dd, $\left.\mathrm{J}=5.4,17.4 \mathrm{~Hz}, 4-\mathrm{H}_{\text {trans }}\right), 3.76(3 \mathrm{H}, \mathrm{s}, \mathrm{MeO}), 4.01\left(1 \mathrm{H}, \mathrm{dd}, \mathrm{J}=11.8,17.4 \mathrm{~Hz}, 4-\mathrm{H}_{\text {cis }}\right), 5.59(1 \mathrm{H}$, dd, J = 5.4, 11.8 Hz, 3-H), 6.88-8.12 (m, 12 arom. H), 8.57 (1H, s, 4'-H), 12.36 (1H, s, COOH); ${ }^{13} \mathrm{C}-\mathrm{NMR}\left(\delta, \mathrm{DMSO}_{\mathrm{d}}\right.$ ): 44.6, 55.0, 61.9, 112.4, 114.4, 115.9, 119.1, 119.6, 120.5, 124.8, 126.9, $128.9,130.7,132.2,133.4,139.6,145.7,146.5,153.1,158.6,167.1$; IR $\left(\mathrm{cm}^{-1}\right): 1726,1672,1599$, $1514,1432,1395,1307,1286,1244,1173,1127,1027,975,871,835$, 752; Anal. Calcd. for $\mathrm{C}_{26} \mathrm{H}_{20} \mathrm{~N}_{2} \mathrm{O}_{5}$ : C, 70.90; H, 4.58; N, 6.36. Found: C, 70.96; H, 4.63; N, 6.28\%.

1-(4-Carboxyphenyl)-3-(3-coumarinyl)-5-(4-fluorophenyl)-2-pyrazoline (19). Isolated as red needles in 69\% yield, mp 296-298 ${ }^{\circ} \mathrm{C}$ (methanol); ${ }^{1} \mathrm{H}-\mathrm{NMR}\left(\delta, \mathrm{DMSO}-\mathrm{d}_{6}\right): 3.28(1 \mathrm{H}, \mathrm{dd}, \mathrm{J}=5.9$, $\left.17.8 \mathrm{~Hz}, 4-\mathrm{H}_{\text {trans }}\right), 4.01\left(1 \mathrm{H}, \mathrm{dd}, \mathrm{J}=12.1,17.8 \mathrm{~Hz}, 4-\mathrm{H}_{\text {cis }}\right), 5.70(1 \mathrm{H}, \mathrm{dd}, \mathrm{J}=5.9,12.1 \mathrm{~Hz}, 3-\mathrm{H})$, 7.06-8.11 (m, 12 arom. H), $8.54\left(1 \mathrm{H}, \mathrm{s}, 4{ }^{\prime}-\mathrm{H}\right), 12.41(1 \mathrm{H}, \mathrm{s}, \mathrm{COOH}) ;{ }^{13} \mathrm{C}-\mathrm{NMR}\left(\delta, \mathrm{DMSO}-\mathrm{d}_{6}\right)$ : $44.5,61.6,112.9,115.5,115.9,116.1,119.4,120.5,124.8,125.3,127.8,128.6,128.9,130.8$, 132.3, 139.7, 142.9, 147.1, 150.2, 158.2, 166.7; IR (cm $\left.{ }^{-1}\right): 1733,1676,1599,1513,1416,1370$, 1313, 1287, 1228, 1174, 1132, 877, 834, 754; Anal. Calcd. for $\mathrm{C}_{25} \mathrm{H}_{17} \mathrm{FN}_{2} \mathrm{O}_{4}$ : C, 70.09; H, 4.01; N, 6.54. Found: C, 70.14; H, 4.05; N, 6.61\%.

1-(4-Carboxyphenyl)-5-(4-chlorophenyl)-3-(3-coumarinyl)-2-pyrazoline (20). Prepared as red needles in 73\% yield, mp 271-273 ${ }^{\circ} \mathrm{C}$ (methanol); ${ }^{1} \mathrm{H}-\mathrm{NMR}\left(\delta, \mathrm{DMSO}-\mathrm{d}_{6}\right): 3.25(1 \mathrm{H}, \mathrm{dd}, \mathrm{J}=$ $\left.5.1,17.6 \mathrm{~Hz}, 4-\mathrm{H}_{\text {trans }}\right), 4.02\left(1 \mathrm{H}, \mathrm{dd}, \mathrm{J}=12.0,17.6 \mathrm{~Hz}, 4-\mathrm{H}_{\text {cis }}\right), 5.67(1 \mathrm{H}, \mathrm{dd}, \mathrm{J}=5.1,12.0 \mathrm{~Hz}, 3-$ $\mathrm{H}), 7.08-8.12$ (m, 12 arom. H), $8.56\left(1 \mathrm{H}, \mathrm{s}, 4{ }^{\prime}-\mathrm{H}\right), 12.48(1 \mathrm{H}, \mathrm{s}, \mathrm{COOH}) ;{ }^{13} \mathrm{C}-\mathrm{NMR}(\delta$, DMSO$\left.\mathrm{d}_{6}\right)$ : 44.5, 61.7, 112.4, 115.9, 117.4, 119.2, 119.5, 120.8, 124.8, 127.6, 128.7, 129.1, 130.6, 130.8, $131.1,132.6,139.8,142.9,145.8,146.3,153.1,158.0,167.1$; IR $\left(\mathrm{cm}^{-1}\right): 1730,1671,1600,1517$, $1488,1420,1395,1310,1292,1222,1175,1181,977,870,834,762$; Anal. Calcd. for $\mathrm{C}_{25} \mathrm{H}_{17} \mathrm{ClN}_{2} \mathrm{O}_{4}$ : C, 67.49; H, 3.85; N, 6.29. Found: C, 67.41; H, 3.89; N, 6.36\%.

5-(4-Bromophenyl)-1-(4-carboxyphenyl)-3-(3-coumarinyl)-2-pyrazoline (21). Isolated as red needles in 65\% yield, mp 298-300 ${ }^{\circ} \mathrm{C}$ (methanol); ${ }^{1} \mathrm{H}-\mathrm{NMR}\left(\delta, \mathrm{DMSO}-\mathrm{d}_{6}\right): 3.29(1 \mathrm{H}, \mathrm{dd}, \mathrm{J}=5.3$, $\left.18.0 \mathrm{~Hz}, 4-\mathrm{H}_{\text {trans }}\right), 4.06\left(1 \mathrm{H}, \mathrm{dd}, \mathrm{J}=11.7,18.0 \mathrm{~Hz}, 4-\mathrm{H}_{\text {cis }}\right), 5.69(1 \mathrm{H}, \mathrm{dd}, \mathrm{J}=5.3,11.7 \mathrm{~Hz}, 3-\mathrm{H})$, 7.08-8.14 (m, 12 arom. H), $8.56\left(1 \mathrm{H}, \mathrm{s}, 4{ }^{\prime}-\mathrm{H}\right), 12.48(1 \mathrm{H}, \mathrm{s}, \mathrm{COOH}) ;{ }^{13} \mathrm{C}-\mathrm{NMR}\left(\delta, \mathrm{DMSO}-\mathrm{d}_{6}\right)$ : $44.4,61.7,112.4,115.9,119.2,119.5,120.8,121.5,124.8,128.6,130.5,130.7,131.6,132.0$, $132.3,139.8,140.8,145.8,146.3,153.1,158.0,167.1$; IR $\left(\mathrm{cm}^{-1}\right): 1729,1671,1599,1517,1486$, 
1422, 1396, 1309, 1290, 1222, 1175, 1131, 871, 761; Anal. Calcd. for $\mathrm{C}_{25} \mathrm{H}_{17} \mathrm{BrN}_{2} \mathrm{O}_{4}$ : C, 61.36; H, 3.50; N, 5.72. Found: C, 61.45, H, 3.55; N, 5.67\%.

\section{Acknowledgements}

The present study was sponsored by the Hungarian National Research Fund (Grant No. OTKA T049468) for which our gratitude is expressed. Technical assistance of Mrs. M. Nagy is highly appreciated.

\section{References}

1. Geissman, T. A., The Chemistry of Flavonoid Compounds, Pergamon Press: Oxford, 1962.

2. Harborne, J. B. The Flavonoids: Advances in Research since 1986, Chapman and Hall: London, 1994.

3. Murray, D. H.; Mendez, J.; Brown, S. A. The Natural Coumarins: Occurrence, Chemistry and Biochemistry, J. Wiley and Sons: New York, 1982.

4. Miky, J. A. A.; Farrag, A. A. Indian J. Chem. 1997, 36B, 357.

5. Brahmbhatt, D. I.; Raolji, G. B.; Pandya, S. U.; Pandya, U. R. Indian J. Chem. 1999, 38B, 212.

6. Essawy, A. I.; Elkady, M.; Mohamed, A. Y. Indian J. Chem. 1980, 19B, 567.

7. van den Goorbergh, J. A. M.; van der Steeg, M.; van der Gen, A. Synthesis 1984, 859.

8. Dimitrova, E.; Anghelova, Y. Synth. Commun. 1986, 16, 1195.

9. van den Goorbergh, J. A. M.; van der Steeg, M.; van der Gen, A. Synthesis 1987, 314.

10. Yagodinets, P. I.; Skripskaya, O. V.; Chernyuk, I. N.; Shevchuk, M. I. Zh. Obschch. Khim. 1991, 61, 1856; Chem. Abstr. 1992, 116, 194440.

11. Ji, M.; Hu, J.; Hua, W.; Hu, H. Indian J. Chem. 2001, 40B, 1223.

12. Djudjic, R.; Trkovnik, M. Croat. Chem. Acta 1990, 63, 13.

13. Prashant, A.; Srinivas Rao, S.; Chowdary, K. S.; Krishnan, V. S. A. Heterocycl. Commun. 2001, 7, 61 .

14. Mulwaad, V. V.; Pawar, R. B. Indian J. Chem. 2003, 42B, 2091.

15. Lévai, A.; Tóth, G.; Gondos, T.; Jekö, J.; Brahmbhatt, D. I. Heterocycles 2006, 68, 1319.

16. Lévai, A.; Jekő, J.; Gondos, T.; Simon, A.; Tóth, G. J. Heterocycl. Chem. 2007, 44, 1453.

17. Lévai, A.; Jekő, J.; Brahmbhatt, D. I. J. Heterocycl. Chem. 2005, 42, 1231.

18. Brown, R.E.; Shavrel, Jr., J. US Patent 1972, 3,624,102; Chem. Abstr. 1972, 76, 59618.

19. Lombardino, J. G.; Otternes, I. G. J. Med. Chem. 1981, 24, 830.

20. Ramalingham, K.; Thyvekikakath, G. X.; Berlin, K. D.; Chesnut, R. W.; Brown, R. A.; Durham, N. N.; Ealick, S. E.; van der Helm, D. J. Med. Chem. 1977, $20,847$. 
21. Elguero, J. in Comprehensive Heterocyclic Chemistry II, Katritzky, A. R.; Rees, C. W., Scriven, E. F. V., Eds., Pergamon Press: Oxford, 1996, Vol. 3, p1.

22. Lévai, A. Khim. Geterotsikl. Soedin. 1997, 747.

23. Lévai, A. J. Heterocycl. Chem. 2002, 39, 1.

24. Lévai, A.; Szöllősy, Á.; Tóth, G. J. Chem. Research (S) 1985, 392.

25. Tóth, G.; Szöllősy, Á.; Lóránd, T.; Kónya, T.; Szabó, D.; Földesi, A.; Lévai, A. J. Chem. Soc. Perkin Trans. 2 1989, 319.

26. Szöllősy, Á.; Tóth, G.; Lóránd, T.; Kónya, T.; Aradi, F.; Lévai, A. J. Chem. Soc. Perkin Trans. 2 1991, 489.

27. Lévai, A. J. Heterocycl. Chem. 1998, 35, 13.

28. Lévai, A. Heterocycl. Commun. 1999, 5, 151.

29. Lévai, A.; Patonay, T.; Silva, A. M. S.; Pinto, D. C. G. A., Cavaleiro, J. A. S. J. Heterocycl. Chem. 2002, 39, 751.

30. Lévai, A. Heterocycl. Commun. 2003, 9, 287.

31. Lévai, A.; Silva, A. M. S.; Pinto, D. C. G. A.; Cavaleiro, J. A. S.; Alkorta, I.; Elguero, J.; Jekö, J. Eur. J. Org. Chem. 2004, 4672.

32. Mishriky, N.; Ibrahim, Y. A.; Girgis, A. S.; Fawzy, N. G. Pharmazie 1999, 54, 738.

33. Zampieri, D.; Mamolo, M. G.; Laurini, E.; Scialino, G.; Banfi, E.; Vio, L. Bioorg. Med. Chem. 2008, 16, 4516.

34. Chimenti, F.; Bizzarri, B.; Manna, F.; Bolasco, A.; Secci, D.; Chimenti, P.; Granese, A.; Rivanera, D.; Lilli, D.; Scaltrito, M. M.; Benciaglia, M. I. Bioorg. Med. Chem. Lett. 2005, 15, 603 .

35. Lóránd, T.; Szabó, D.; Földesi, A.; Párkányi, L.; Kálmán, A.; Neszmélyi, A. J. Chem. Soc. Perkin Trans. 1 1985, 481.

36. Lóránd, T.; Aradi, F.; Szöllősy, Á.; Tóth, G.; Kónya, T. Monatsh. Chem. 1996, 127, 971.

37. Jagtap, P. G.; Degterev, A.; Choi, S.; Keys, H.; Yuan, J.; Cuny, G. D. J. Med. Chem. 2007, 50, 1886.

38. Kudar, V.; Zsoldos-Mády, V.; Simon, K.; Csámpai, A.; Sohár, P. J. Organometallic Chem. 2005, 690, 4018.

39. Lévai, A.; Jekő, J. J. Heterocycl. Chem. 2006, 43, 1303.

40. Lévai, A.; Jekö, J. ARKIVOC 2007, (i), 134.

41. Lévai, A.; Jekö, J. Acta Chim. Slov., in press. 